\title{
SOME APPROXIMATION PROPERTIES OF MODIFIED BASKAKOV STANCU OPERATORS
}

\author{
Anshul Srivastava ${ }^{1}$ \\ ${ }^{1}$ Assistant professor, Department of Applied Science and Humanities, Northern India Engineering \\ College,Indraprastha University, New Delhi, India.
}

\begin{abstract}
In the present paper, we prove a global direct theorem for the modified Baskakovstancu operators in terms of Ditzian-Totik modulus of smoothness. Here, we have modified our operators by taking weight function of Beta operators and then generalizing it as stancu type generalized operators. We will also see that taking weight function of Beta operators will give better approximation. We study a global direct theorem using simultaneous approximation for ourstancu type generalized operator in $L_{p}[0, \infty)$. Here, first we estimate recurrence relation for moments and then develop some global direct results by making our stancu type generalized operators positive using differential and integral operators. In this paper, our effort is to give better global approximation for our stancu type generalized operator than the earlier integral modifications of Baskakov operators studied by various authors. Here, we will extend our results for the whole interval $[0, \infty)$. In this paper, we will also make use of the fact that second modulus of smoothness introduced by Ditzian-Totik is equivalent to modified $k$-functional and $L_{p}^{r}[0, \infty)$ is not contained in $L_{1}^{r}[0, \infty)$ for obtaining results. Here, Riesz-Thorin theorem and Leibnitz theorem is used extensively for doing simultaneous approximation. We have also used Fubini's theorem for obtaining results.
\end{abstract}

Key words: Stancu type generalization, simultaneous approximation, modulus of smoothness.

\section{INTRODUCTION}

Several authors have defined and studied different modifications of Baskakov operators. V.Gupta [1] modified Baskakov operators by taking weight function of Beta operators on $L_{1}[0, \infty)$ as,

$G_{(n)}(f, x)=\sum_{m=0}^{\infty} k_{(n, m)}(x) \int_{0}^{\infty} b_{(n, m)}(t) f(t) d t \forall x \in[0, \infty)$

where $k_{(n, m)}(x)=\left(\begin{array}{c}n+m-1 \\ m\end{array}\right) \frac{x^{k}}{(1+x)^{(n+k)}}$

$\operatorname{and} b_{(n, m)}(t)=\frac{t^{m}}{(1+t)^{(n+m+1)} B(m+1, n)}$

Here, $B(m+1, n)=\frac{m !(n-1) !}{(n+m) !}$ is Beta function .

In the present paper, we will study global direct theorem in simultaneous approximation for Stancu type generalization [2] of above modifiedBaskakov operators defined as,

$G_{(n)}^{(\alpha, \beta)}(f, x)=\sum_{m=0}^{\infty} k_{(n, m)}(x) \int_{0}^{\infty} b_{(n, m)}(t) f\left(\frac{n t+\alpha}{n+\beta}\right) d t$

Here, for $\alpha=0, \beta=0$ the above operator reduces to (1.1).

Ditzian-Totik [3] defined second order modulus of smoothness as,

$\omega_{\varphi(x)}^{2}(f, t)_{p}=\sup _{0 \leq h \leq t}\left\|\Delta_{h \varphi(x)}^{2} f(x)\right\|_{p}$ where $\varphi(x)=\frac{1}{\{x(1+x)\}^{-1 / 2}}$

and $\Delta_{h}^{2} f(x)=$

$\{f(x-h)-2 f(x)+f(x+h), \quad$ if $|x-h, x+h| \subset[0, \infty)$ otherwise.

It may be noted that,

$$
L_{p}^{r}[0, \infty) \not \subset L_{1}^{r}[0, \infty)
$$

where $L_{1}^{r}[0, \infty)$ denotes the class of functions $g$ and is defined as,

$$
\begin{aligned}
& L_{1}^{r}[0, \infty):= \\
& \left\{g: g^{(r)} \in L_{1}[0, \infty), \forall \alpha \in[0, \infty) \text { and }\left|g^{(r)}(t)\right| \leq \frac{D}{(1+t)^{-d}}\right\}
\end{aligned}
$$

Here, $D$ and $d$ are constants depending on $g$.

Throughout the paper we denote by $C$ the positive constants not necessarily the same at each occurrence.

\section{SOME DEFINITIONS AND RESULTS.}

\section{Definition 2.1}

For every $n \in N$ and $n>(r+1)$, we have,
a) $\sum_{m=0}^{\infty} K_{(n, m)}(x)=1$.
b) $\int_{0}^{\infty} b_{(n, m)}(t) d t=1$. 

c) $m k_{(n, m)}(x)=n k_{(n+1, m-1)}(x)$.
d) $\int_{0}^{\infty} t b_{(n-r, m+r)}(t) d t=$ $\{(m+r+1) /(n-r-1)\}$.

Lemma 2.2.[1] Let $q, r \in N_{0}$, we define, $q$ th moment as,

$T_{(r, n, q)}^{(\alpha, \beta)}(x)=\sum_{m=0}^{\infty} k_{(n+r, m)}(x) \int_{0}^{\infty} b_{(n-r, m+r)}(t)\left(\frac{n t+\alpha}{n+\beta}-x\right)^{q} d t$

Here, for each $x \in[0, \infty)$ and $\alpha=0, \beta=0$,

$T_{(r, n, q)}(x)=O\left(n^{-[(q+1) / 2]}\right)$

where $[\gamma]$ denotes integral part of $\gamma$.

Also for $\alpha=0, \beta=0$, we have [6] [7],

$T_{(r, n, 2 q)}(x)=\sum_{i=0}^{s} q_{(i, q, n)}(x)\left[\varphi^{2}(x) / n\right]^{q-i}\left(\frac{1}{n^{2 i}}\right)$

$T_{r, n, 2 q+1}(x)=$

$(1+2 x) \sum_{i=0}^{q} u_{(i, q, n)}(x)\left[\varphi^{2}(x) / n\right]^{q-i}\left(\frac{1}{n^{(2 i+1)}}\right)$

Here, $q_{(i, q, n)}(x)$ and $u_{(i, q, n)}(x)$ are polynomials in $x$ of fixed degree with coefficients that are bounded uniformly for all $n$.

Proof.The proof of above lemma easily follows along the lines of [4], [5].

Lemma 2.3.If $f \in L_{p}^{r}[0, \infty) \cup L_{1}^{r}[0, \infty), 1 \leq p \leq \infty, n>$ $\frac{r}{(1+s)^{-1}}$ and $x \in[0, \infty)$, then,

$G_{(n)}^{(\alpha, \beta)}\left(f^{(r)}, x\right)$

$=\mu_{(n, r)} \sum_{m=0}^{\infty} k_{(n+r, m)}(x) \int_{0}^{\infty} b_{(n-r, m+r)}(t) f^{(r)}\left(\frac{n t+\alpha}{n+\beta}\right) d t$

Where,

$$
\begin{array}{r}
\mu_{(n, r)}=(n+r-1) !(n-r-1) !((n-1) !)^{-2} \\
=\prod_{l=0}^{r-1}(n+l)\{n-(l+1)\}^{-1}
\end{array}
$$

Proof.Using Leibnitz theorem, we have ,

$$
\begin{aligned}
G_{(n)}^{(\alpha, \beta)}\left(f^{(r)}, x\right)= & \sum_{i=0}^{r} \sum_{m=i}^{\infty}\left(\begin{array}{l}
r \\
i
\end{array}\right) \frac{(n+m+r-i-1) !}{(n-1) !(m-i) !} \\
& \times \frac{(-1)^{r-i} x^{m-i}}{(1+x)^{n+m+r-i}} \\
& \times \int_{0}^{\infty} b_{(n, m)}(t) f\left(\frac{n t+\alpha}{n+\beta}\right) d t
\end{aligned}
$$

$$
\begin{aligned}
& =\frac{(n+r-1) !}{(n-1) !} \sum_{m=0}^{\infty} k_{(n+r, m)}(x) \\
& \times \int_{0}^{\infty} \sum_{i=0}^{r}(-1)^{r-i}\left(\begin{array}{l}
r \\
i
\end{array}\right) b_{(n, m+i)}(t) f\left(\frac{n t+\alpha}{n+\beta}\right) d t .
\end{aligned}
$$

Using again Leibnitz theorem, we have,

$$
\begin{aligned}
& b_{(n-r, m+r)}^{(r)}(t)= \\
& (n-1) !\{(n-r-1) !\}^{-1} \sum_{i=0}^{r}(-1)^{r}\left(\begin{array}{l}
r \\
i
\end{array}\right) b_{(n, m+i)}(t) .
\end{aligned}
$$

Hence,

$$
\begin{aligned}
& G_{(n)}^{(\alpha, \beta)}\left(f^{(r)}, x\right) \\
& =\mu_{(n, r)} \sum_{m=0}^{\infty} k_{(n+r, m)}(x) \int_{0}^{\infty}(-1)^{r} b_{(n-r, m+r)}^{(r)}(t) f\left(\frac{n t+\alpha}{n+\beta}\right) d t .
\end{aligned}
$$

On integrating $r$ times by parts, we get the required result. Here, we see that,operators defined by (2.5)are not positive. To make the operators positive, we introduce the operator,

$G_{(n, r)}^{(\alpha, \beta)} f \equiv D^{r} G_{(n)}^{(\alpha, \beta)} I^{r} f, \quad f \in L_{p}[0, \infty) \cup L_{1}[0, \infty)$,

where $D$ and $I$ are differential and integral operators respectievely.

Therefore we define the operators by,

$G_{(n, r)}^{(\alpha, \beta)}(f, x)=$

$\mu_{(n, r)} \sum_{m=0}^{\infty} k_{(n+r, m)}(x) \int_{0}^{\infty} b_{(n-r, m+r)} f\left(\frac{n t+\alpha}{n+\beta}\right) d t$,

$f \in L_{p}[0, \infty) \cup L_{1}[0, \infty), \quad n>r(1+m)$,

The operators $G_{(n, r)}^{(\alpha, \beta)}$ are positive and the estimation $\left\|G_{(n)}^{(\alpha, \beta)} f^{(r)}-f^{(r)}\right\|_{p}, f \in L_{p}^{r}[0, \infty) \quad$ is equivalent to $\left\|G_{(n, r)}^{(\alpha, \beta)} f-f\right\|_{p}, f \in L_{p}[0, \infty)$.

We can also very easily prove that for $n>(r+1)$,

$\left\|G_{(n, r)}^{(\alpha, \beta)} f\right\|_{1} \leq C\|f\|_{1}$, for $f \in L_{1}[0, \infty)$ and

$\left\|G_{(n, r)}^{(\alpha, \beta)} f\right\| \leq C\|f\|_{\infty}$, for $f \in L_{\infty}[0, \infty)$.

Using Riesz-Thorin theorem, we get,

$\left\|G_{(n, r)}^{(\alpha, \beta)} f\right\|_{p} \leq C\|f\|_{p}$, for $1 \leq p \leq \infty, n>(r+1)$.

Corollary 2.4.For every $q \in N_{0}, n>(r+2 q+1)$ and $x \in[0, \infty)$, we have,

$\left|G_{(n, r)}^{(\alpha, \beta)}\left(\left(\frac{n t+\alpha}{n+\beta}-x\right)^{2 q}, x\right)\right| \leq \frac{C}{n^{q}}\left(\varphi^{2}(x)+\left(\frac{1}{n}\right)\right)^{q}$ and 
$\left|G_{(n, r)}^{(\alpha, \beta)}\left(\left(\frac{n t+\alpha}{n+\beta}-x\right)^{2 q+1}, x\right)\right| \leq$

$C(1+2 x) n^{-(q+1)}\left(\varphi^{2}(x)+\left(\frac{1}{n}\right)\right)^{q}$

where the constant $C$ is independent of $n$.

Also, for fixed $x \in[0, \infty)$ we obtain,

$\left|G_{(n, r)}^{(\alpha, \beta)}\left(\left(\frac{n t+\alpha}{n+\beta}-x\right)^{q}, x\right)\right|=O\left(n^{-[(q+1) / 2]}\right) \quad, \quad n \rightarrow \infty$.

Proof.The estimate (2.6) easily follows from (2.3) and (2.4) along the lines of [6], and (2.7) immediately follows from

Lemma 2.5.Let $t \in[0, \infty)$ and $n>(r+q)$ then,

$G_{(n, r)}^{(\alpha, \beta)}\left(\left(1+\left(\frac{n t+\alpha}{n+\beta}\right)\right)^{-q}, x\right) \leq \frac{c}{(1+x)^{q}}$, for $x \in[0, \infty)$

where the constant $C$ is independent of $n$.

Proof. It is easily verified that,

$\left(1+\left(\frac{n t+\alpha}{n+\beta}\right)\right)^{-q} b_{(n-r, m+r)}(t)=$

$\prod_{l=0}^{q-1} \frac{(n-r+l)}{(n+l+m+1)} b_{(n-r+q, m+r)}(t)$ and

$k_{(n+r, m)}(x)=\frac{1}{(1+x)^{q}} \prod_{l=1}^{q} \frac{(n+r-l+m)}{(n+r-l)} k_{(n+r-q, m)}(x)$.

Using above identities, we get,

$$
\begin{aligned}
& G_{(n, r)}^{(\alpha, \beta)}\left(\left(1+\left(\frac{n t+\alpha}{n+\beta}\right)\right)^{-q}, x\right) \\
= & \mu_{(n, r)} \sum_{m=0}^{\infty} k_{(n+r, m)}(x) \int_{0}^{\infty} b_{(n-r, m+r)}(t)(1 \\
\left.+\left(\frac{n t+\alpha}{n+\beta}\right)\right)^{-q} d t & \mu_{(n, r)} \sum_{m=0}^{\infty} k_{(n+r, m)}(x) \prod_{l=0}^{q-1} \frac{(n-r+l)}{(n+l+m+1)} \\
= & \mu_{(n, r)}^{\infty} \sum_{m=0}^{\infty} \frac{k_{(n+r-q, m)}(x)}{(1+x)^{q}} \prod_{l=1}^{q} \frac{(n+r-l+m)}{(n+r-l)} \\
\times \prod_{l=0}^{q-1} \frac{(n+r-l)}{(n+l+m+1)} & \\
\leq C \frac{1}{(1+x)^{q}} \sum_{m=0}^{\infty} & k_{(n+r-q, m)}(x) \\
& \times \frac{1}{(1+x)^{q}}
\end{aligned}
$$

For $x \in[0, \infty), n \rightarrow \infty$, and monomials $e_{0}, e_{1}$, we have,

$G_{(n, r)}^{(\alpha, \beta)}\left(e_{0}, x\right)=1+O\left(n^{-1}\right)$

$G_{(n, r)}^{(\alpha, \beta)}\left(e_{1}, x\right)=x$

$0 \quad\left(n^{-1}\right)$

(2.9)

Lemma 2.6. For $H_{(n)}^{(\alpha, \beta)}$ given by,

$H_{(n)}^{(\alpha, \beta)}(u)$

$=\left\{\int_{0}^{\infty} \int_{0}^{u}-\int_{0}^{u} \int_{0}^{\infty}\right\} \sum_{m=0}^{\infty} k_{(n+r, m)}(x) b_{(n-r, m+r)}(t)(u$

$\left.-\left(\frac{n t+\alpha}{n+\beta}\right)\right) d t d x$

We have $H_{(n)}^{(\alpha, \beta)}(u) \leq C n^{-1} \varphi^{2}(u)$, where $C$ is a constant independent of $n$.

Proof.The proof of above lemma easily follows along the lines of [7].

\section{DIRECT RESULT}

Theorem 3.1.Suppose $f \in L_{p}[0, \infty), 1 \leq p \leq \infty, n>$ $(r+5)$ then we have,

$$
\left\|G_{(n, r)}^{(\alpha, \beta)} f-f\right\|_{p} \leq C\left\{\omega_{\varphi}^{2}\left(f, \frac{1}{\sqrt{n}}\right)+\left(\frac{1}{n}\right)\|f\|_{p}\right\}
$$

Where the constant $C$ is independent of $n$.

Proof.Using Taylor's expansion, we have,

$g\left(\frac{n t+\alpha}{n+\beta}\right)=g(x)+\left(\left(\frac{n t+\alpha}{n+\beta}\right)-x\right) g^{\prime}(x)+\int_{x}^{t}\left(\left(\frac{n t+\alpha}{n+\beta}\right)-\right.$

$u g^{\prime \prime} u d u$.

Since, $G_{(n, r)}^{(\alpha, \beta)}(f, x)$ are uniformly bounded operators so for every $g \in \bar{W}_{p}^{2}(\varphi,[0, \infty))$, we have,

$$
\left\|G_{(n, r)}^{(\alpha, \beta)} f-f\right\|_{p} \leq C\|f-g\|_{p}+\left\|G_{(n, r)}^{(\alpha, \beta)} g-g\right\|_{p}
$$

Now using (2.6), (2.9), (3.1) and [2], we have,

$$
\begin{aligned}
& \left\|G_{(n, r)}^{(\alpha, \beta)} g-g\right\|_{p} \leq C\left\{\|g\|_{p}+\left\|g^{\prime}\right\|_{L_{p}[0,1]}\right\} \\
& +\left\|(1+2 x) g^{\prime}\right\|_{L_{p}[1, \infty)} \\
& +\left\|G_{(n, r)}^{(\alpha, \beta)}(R(g, t, x), x)\right\|_{p} \\
& \leq C\left(\frac{1}{n}\right)\left[\|g\|_{p}+\left\|\varphi^{2} g^{\prime \prime}\right\|_{p}\right]+\left\|G_{(n, r)}^{(\alpha, \beta)}(R(g, t, x), x)\right\|_{p} \\
& \text { where } R(g, t, x)=\int_{x}^{t}\left(\left(\frac{n t+\alpha}{n+\beta}\right)-u\right) g^{\prime \prime}(u) d u
\end{aligned}
$$


Now, we will prove this,

$\left\|G_{(n, r)}^{(\alpha, \beta)}(R(g, t, x), x)\right\|_{p} \leq C\left(\frac{1}{n}\right)\left\|\left(\varphi^{2}+\left(\frac{1}{n}\right)\right) g^{\prime \prime}\right\|_{p} \quad$ for cases $\boldsymbol{p}=\infty$ and $\boldsymbol{p}=1$.

The cases $1 \leq \boldsymbol{p} \leq \infty$ follows again by Riesz-Thorin theorem.

Case 1: Using (2.6) for the case $\boldsymbol{m}=1$ and Lemma 4, the case $\boldsymbol{p}=\infty$ easily follows [5].

Case 2: For $\boldsymbol{p}=1$, we will use Fubini's theorem twice, the definition of $\boldsymbol{H}_{(\boldsymbol{n})}^{(\boldsymbol{\alpha}, \boldsymbol{\beta})}(\boldsymbol{u})$ and Lemma 5 as,

$$
\begin{aligned}
& \int_{0}^{\infty}\left|G_{(n, r)}^{(\alpha, \beta)}(R(g, t, x), x)\right| \\
& \leq \mu_{(n, r)} \int_{0}^{\infty} \sum_{m=0}^{\infty} k_{(n+r, m)}(x) \int_{0}^{\infty} b_{(n-r, m+r)}(t) \mid \int_{x}^{t}\left(\left(\frac{n t+\alpha}{n+\beta}\right)\right. \\
& -u) g^{\prime \prime}(u) \mid d t d x \\
& =\mu_{(n, r)} \int_{0}^{\infty}\left|g^{\prime \prime}(u)\right|\left\{\int_{0}^{\infty} \int_{0}^{u}-\int_{0}^{u} \int_{0}^{\infty}\right\}\left(u-\left(\frac{n t+\alpha}{n+\beta}\right)\right) \\
& \times \sum_{m=0}^{\infty} k_{(n+r, m)}(x) b_{(n-r, m+r)}(t) d t d x d u \\
& =\mu_{(n, r)} \int_{0}^{\infty}\left|g^{\prime \prime}(u)\right| H_{(n)}^{(\alpha, \beta)}(u) d u \\
& \leq C\left(\frac{1}{n}\right)\left\|\varphi^{2} g^{\prime \prime}\right\|_{1} \\
& \leq C\left(\frac{1}{n}\right)\left\|\left(\varphi^{2}+\left(\frac{1}{n}\right)\right) g^{\prime \prime}\right\|
\end{aligned}
$$

where $\boldsymbol{C}$ is independent of $\boldsymbol{n}$. Hence (3.4) holds by RieszThorin theorem for $1 \leq \boldsymbol{p} \leq \infty$. Combining the estimates of (3.2), (3.3) and (3.4) we get,

$$
\begin{aligned}
\left\|G_{(n, r)}^{(\alpha, \beta)} f-f\right\|_{p} & =C\|f-g\|_{p} \\
& +C\left(\frac{1}{n}\right)\left\{\|f-g\|_{p}+\left\|\varphi^{2} g^{\prime \prime}\right\|_{p}\right. \\
& \left.+\left\|\left(\varphi^{2}+\left(\frac{1}{n}\right)\right) g^{\prime \prime}\right\|_{p}\right\}
\end{aligned}
$$

$\leq C\left\{\|f-g\|_{p}+\left(\frac{1}{n}\right)\left\|\varphi^{2} g^{\prime \prime}\right\|_{p}+\left(\frac{1}{n^{2}}\right)\left\|g^{\prime \prime}\right\|_{p}+\right.$

1nfp.

Now, we take infimum over all $\boldsymbol{g} \in \overline{\boldsymbol{W}}_{\boldsymbol{p}}^{2}(\boldsymbol{\varphi},[0, \infty))$ on the right hand side, we get,

$$
\left\|G_{(n, r)}^{(\alpha, \beta)} f-f\right\|_{p} \leq C\left\{\bar{K}_{\varphi}^{2}\left(f,\left(\frac{1}{n}\right)\right)+\left(\frac{1}{n}\right)\|f\|_{p}\right\} .
$$

Hence the result.

Remark.Above theorem is true on the space $\boldsymbol{L}_{p}[0, \infty), 1 \leq$ $\boldsymbol{p} \leq \infty$.

As,

$\lim _{n \rightarrow \infty} \omega_{\varphi}^{2}\left(f, \frac{1}{n}\right)=0$ for all $f \in L_{p}[0, \infty), 1 \leq p \leq \infty$,

We have,

$\lim _{n \rightarrow \infty}\left\|G_{(n, r)}^{(\alpha, \beta)} f-f\right\|_{p}=0$ for every $f \in L_{p}[0, \infty)$.

\section{CONCLUSION}

Our modified Baskakovstancu operator gives better global simultaneous approximation in $\boldsymbol{L}_{\boldsymbol{p}}$ spaces than all other earlier integral modifications ofBaskakov operators. Here, we have obtained global results for whole interval $[0, \infty)$. Recurrence relation for our modified Baskakovstancu operator is different from other modifiedBaskakov operators. Use of Riesz-Thorin theoremhas enabled us to get better approximation results. For all bounded functions $f \in C[0, \infty)$, function $\boldsymbol{f}$ satisfy,

$\lim _{x \rightarrow \infty} f(x)=L_{\infty}<\infty$, if $p=\infty$

\section{ACKNOWLEDGEMENT.}

The author is grateful to the refree for many suggestions that greatly improved this paper.

\section{REFERENCES}

[1] V.Gupta, A note on modified Baskakov operators, Approx. Theory and its Appl. 10(3) (1994), 74-78.

[2] D.D.Stancu, Approximation of function by a new class of polynomial operators, Rev. Roum. Math. Pureset Appl., 13(8) (1968), 1173-1194.

[3] Z.Ditzian and V.Totik. "Moduli of smoothness", Springer Series in computational Mathematics 9, Springer-Verlag, Berlin, Heidelberg, New York, 1987.

[4] A.Sahai and G.Prasad, On simultaneous approximation by modified Lupas operators, J. Approx. Theory 45 (1985), 122-128.

[5] R.P. Sinha, P.N. Agarwal and V.Gupta, On Simultaneous approximation by modified Baskakov operators, Bull. Soc. Math. Belg. Ser. B 42(2) (1991), 217-231.

[6] M.Heilmann, Direct and converse results for operators of Baskakov-Durrmeyer type, Approx. Theory and its Appl. 5(1) (1989), 105-127.

[7] Z.Ditzian and K.Ivanov, Bernstein type operators and their derivatives, J. Approx. Theory 56 (1989), 72-90. 